\title{
Relaciones Biométricas del Anillo Atrioventricular Izquierdo y las Arterias Coronarias en el Hombre
}

\author{
Biometric Relationships Between the Left Atrioventricular Ring and the Coronary Arteries in Man
}

\author{
"Ilse Pradenas; ** Jorge Henriquez \& **Enrique Olave
}

PRADENAS, I.; HENRIQUEZ, J. \& OLAVE, E. Relaciones biométricas del anillo atrioventricular izquierdo y las arterias coronarias en el Hombre. Int. J. Morphol., 27(3):683-689, 2009.

RESUMEN:La relación de las arterias coronarias con los anillos atrioventriculares se torna importante en casos de reemplazo o plastía de la valva correspondiente. La proximidad de la rama circunfleja de la arteria coronaria izquierda al anillo atrioventricular izquierdo, a nivel de la comisura anterior, ha traído complicaciones quirúrgicas, en algunos casos con resultados fatales. Basado en lo anterior, estudiamos las relaciones biométricas entre las arterias coronarias y el anillo atrioventricular izquierdo. Utilizamos 58 corazones de individuos chilenos, 28 fijados en formaldehído al $10 \%$ y 30 frescos de individuos brasileños, de edades comprendidas entre 18 y 84 años, de ambos sexos y sin aparente cirugía cardíaca. Disecamos la región de ambos surcos coronarios, para luego clasificar las piezas según el tipo de dominancia coronaria. Luego, los atrios fueron resecados dejando visibles los anillos atrioventriculares y su relación con las arterias coronarias. Se efectuaron mediciones de la distancia de las arterias coronarias y sus ramas en relación al anillo atrioventricular izquierdo. Se establecieron 5 puntos en la porción posterior del anillo, distribuidos en sentido antihorario. Los puntos 1 y 5 localizados a nivel de las comisuras anterior y posterior, respectivamente. La menor distancia entre las ramas de las arterias coronarias y el anillo atrioventricular izquierdo se presentó en corazones con dominancia coronaria izquierda, y fue de 3,8 mm (corazones fijados) y 3,0 mm (corazones frescos).El tercio proximal de la rama circunfleja, asociado a una mayor proximidad al anillo mitral, constituye el área de mayor predisposición a una lesión iatrogénica durante un reemplazo y/o plastía valvar.

PALABRAS ClAVE: Anatomía; Corazón; Arteria coronaria; Anillo atrioventricular izquierdo; Cirugía valvar.

\section{INTRODUCCIÓN}

La irrigación del corazón procede de las arterias coronarias, así denominadas probablemente a causa del trayecto de su porción inicial que rodea el corazón a modo de corona (Testut \& Latarjet, 1960).

Las arterias coronaria derecha y la rama circunfleja de la arteria coronaria izquierda rodean a los anillos atrioventriculares, presentando durante todo su recorrido una relación directa con las valvas atrioventriculares correspondientes; la rama circunfleja, con una longitud aproximada de $8 \mathrm{~cm}$ (Biscegli et al., 1996).

Las valvulopatías son afecciones que comprometen las valvas cardíacas. Las valvas más comúnmente comprometidas son la mitral y luego la aórtica. Se pueden clasificar de acuerdo al tipo de lesión en estenosis, insuficiencias o dobles lesiones (estenosis + insuficiencia).En aquellos casos de insuficiencia mitral aguda, en que no es posible efectuar una reconstrucción del aparato mitral, debe efectuarse un reemplazo de la valva.

El reemplazo valvar izquierdo se encuentra asociado con ciertos riesgos operatorios, siendo el más serio la lesión a una de las estructuras que se dispone en proximidad íntima con la valva, la rama circunfleja de la arteria coronaria izquierda.

En la zona del anillo atrioventricular, la exploración visual de una lesión puede pasar desapercibida y puede traer como consecuencia una grave lesión vascular. Según Cabrol \& Christides (1976) la ligadura total o parcial de la rama circunfleja puede producir desde isquemia del territorio involucrado hasta necrosis del tejido afectado.

\footnotetext{
* Unidad de Anatomía, Facultad de Ciencias de la Salud, Universidad de Antofagasta, Chile.

${ }^{* *}$ Facultad de Medicina, Universidad de La Frontera, Temuco, Chile.
} 
En el lado izquierdo, la unión anular de la cúspide posterior es paralela a la rama circunfleja de la arteria coronaria izquierda y en el derecho, al seno coronario. Seccombe \& Schaff (1999) señalaron que en el sistema coronario, tanto en el de dominancia izquierda y el balanceado, la rama circunfleja podría a través de su curso ser paralela y muy próxima al anillo atrioventricular posterior.

En estudio realizado por Danielson et al. (1967), se observaron tres casos (de 31) de lesión de la rama circunfleja por suturas de fijación de la prótesis al anillo atrioventricular izquierdo, a 3,0 - 3,5 cm de su origen. La oclusión de la rama circunfleja habitualmente ocurre cuando se colocan suturas demasiado profundas en las proximidades de la comisura anterior.

En consecuencia, existe riesgo de lesión de la arteria circunfleja durante la reparación de la valva atrioventricular izquierda, sobre todo en pacientes con dominancia coronaria izquierda y/o dominancia coronaria balanceada, y por consiguiente, es importante conocer la anatomía coronaria preoperatoriamente (Tavilla \& Pacini, 1998). Por otra parte, Virmani et al. (1982) informaron de otros tres casos de obliteración de la arteria circunfleja durante la cirugía de la valva mitral; dos de ellos presentaron dominancia coronaria izquierda, y el último, dominancia coronaria balanceada. duos chilenos y brasileños adultos, de ambos sexos, con edades comprendidas entre 18 y 84 años. El análisis estadístico se realizó en base a una muestra no probabilística de 58 corazones humanos, 28 corazones fijados previamente en formol (corazones chilenos) y 30 corazones frescos (corazones brasileños). Para describir los datos se utilizaron fundamentalmente métodos gráficos y medidas de tendencia central y de dispersión, realizando la prueba t para muestras independientes, para comparar las distancias de los puntos de medición entre corazones fijados y frescos.

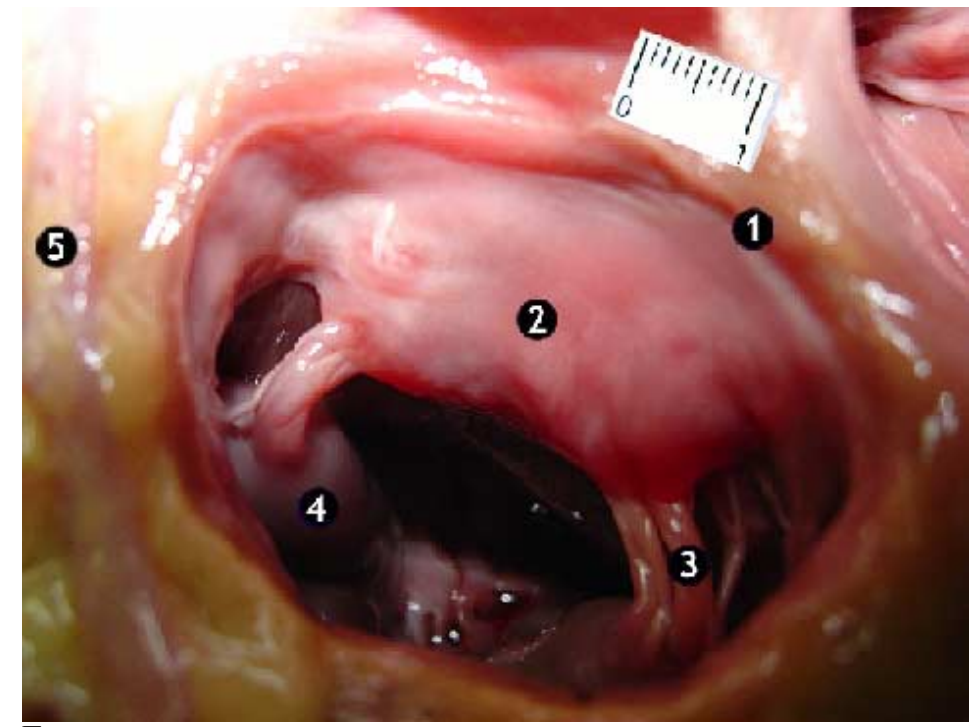

Fig. 1. Corazón fresco, sexo masculino, 48 años. 1. Anillo mitral; 2. Cúspide anterior; 3. Cuerdas tendinosas; 4. Músculo papilar; 5. Rama circunfleja.
Las complicaciones técnicas relacionadas con la implantación de una prótesis mitral se deben también a la proximidad de otras estructuras funcionalmente importantes, que rodean el anillo de la valva, como son: el espacio intervalvar, la rama retroventricular izquierda de la arteria coronaria derecha, las fibras de conducción del fascículo atrioventricular, el seno coronario, la arteria del nodo atrioventricular, la pared posterior del ventrículo izquierdo y la porción membranosa del septo interventricular (Du Plessis \& Marchand, 1964).

Basados en lo anterior, el presente estudio tuvo como objetivo determinar la proximidad de los vasos coronarios respecto de la valva atrioventricular izquierda, considerando su dominancia coronaria, aportando una base de apoyo anatómico a la cirugía cardiaca.

\section{MATERIAL Y MÉTODO}

Se seleccionó una muestra al azar de 58 corazones, sin aparente patología coronaria, correspondientes a indivi-
Se disecó la región del surco coronario, clasificando los corazones según el tipo de dominancia coronaria (Schlessinger, 1940): derecha, izquierda y circulación balanceada. Los atrios fueron resecados, dejando visibles las arterias coronarias y los anillos atrioventriculares (Fig.1).

Se efectuaron mediciones entre las arterias coronarias y el anillo atrioventricular izquierdo, y basados en Cornu et al. (1995) se establecieron 5 puntos en la porción posterior del anillo, distribuidos en sentido antihorario, los puntos 1 y 5 localizados a nivel de las comisuras anterior y posterior, respectivamente; el punto 3 correspondió al punto medio posterior del anillo, los otros dos (puntos 2 y 4) dispuestos en forma equidistante entre los puntos comisurales y el punto 3 (Fig. 2). Las medidas fueron tomadas con un paquímetro digital Hardened.

Las fotografías fueron tomadas con cámara Digital Still SONY, modelo MVC-CD500, resolución de 5,0 megapixeles. Los esquemas fueron trabajados con Photo Editor. 


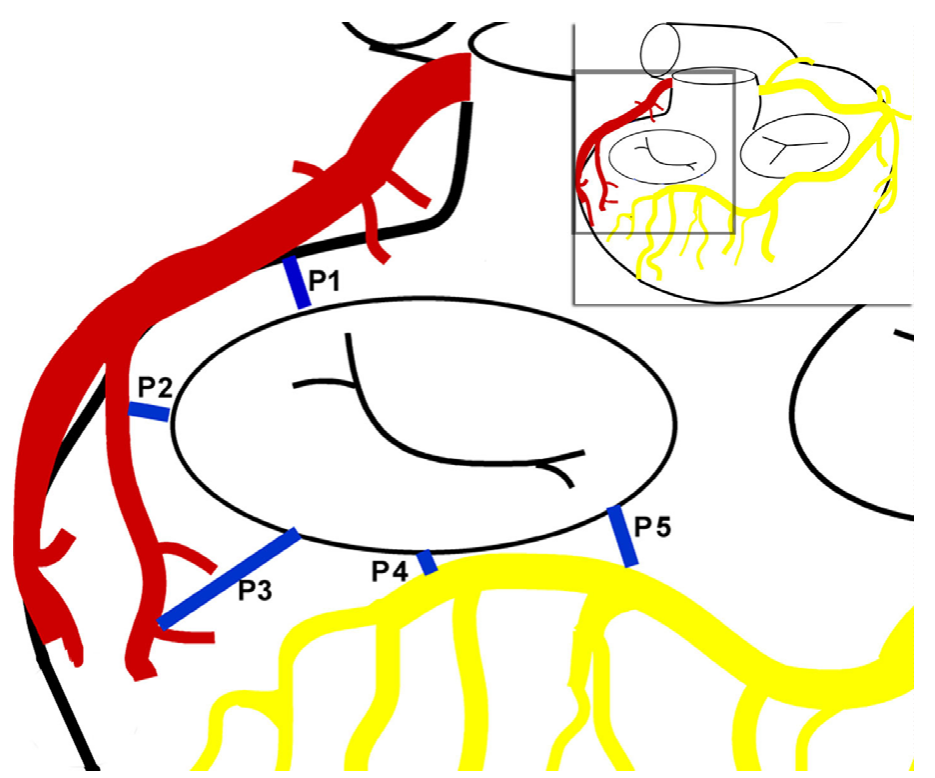

Fig. 2. Representación esquemática de los puntos de medición (P1-P5) entre las arterias coronarias y el anillo atrioventricular izquierdo, basado en Cornu et al. En rojo: Rama circunfleja de la arteria coronaria izquierda; en amarillo: Arteria coronaria derecha.

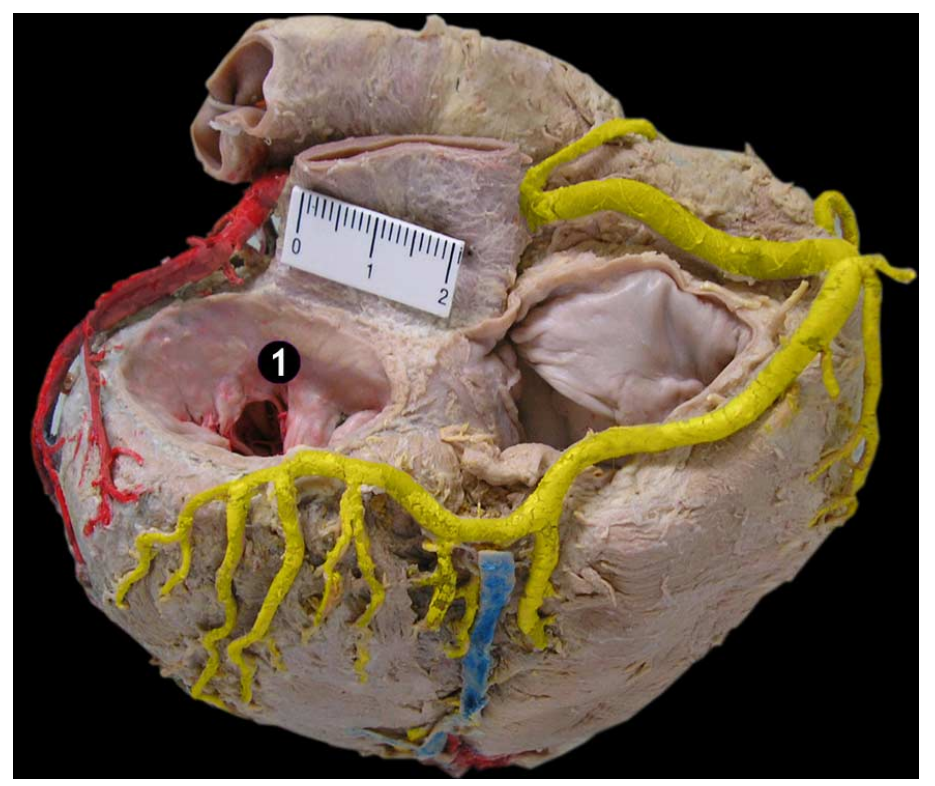

Fig. 3. Dominancia coronaria derecha en corazón humano fijado. En amarillo: arteria coronaria derecha; en rojo: rama circunfleja, 1. Valva mitral.

\section{RESULTADOS}

En la serie estudiada, encontramos $40 \mathrm{ca}-$ sos $(69,0 \%)$ de irrigación coronaria dominante derecha (Fig. 3), 10 casos (17,2 \%) de sistema coronario de dominancia izquierda (Fig. 4) y 8 casos $(13,8 \%)$ de sistema coronario balanceado o codominancia (Fig. 5).

Las distancias entre las arterias coronarias y la porción posterior del anillo atrioventricular izquierdo son presentadas en forma grupal. Se consideraron las siguientes agrupaciones: distancias promedio y desviación estándar de los corazones fijados y frescos (Tablas I y II), diferencia de las distancias promedio entre corazones fijados y frescos (Tabla III), distancia promedio y su respectiva desviación estándar según tipo de dominancia coronaria (Tablas IV y V).

Las diferencias de las distancias promedio entre corazones frescos y fijados indica que en los puntos 1 y 2 los corazones frescos presentaron menor distancia promedio, que corresponde a $-0,53 \mathrm{~mm}$ (valor $\mathrm{p}=0,05)$ y $-0,73 \mathrm{~mm}$ (valor $\mathrm{p}=0,13$ ), respectivamente. En los puntos 3, 4 y 5 las menores distancias promedio corresponden a los corazones fijados con diferencias de $0,14 \mathrm{~mm}$ (valor $\mathrm{p}=0,87$ ), $0,72 \mathrm{~mm}$ (valor $\mathrm{p}=0,31$ ) y de $3,66 \mathrm{~mm}$ (valor $\mathrm{p}=0,0001$ ), respectivamente. Con el nivel de significación de 0,05, la diferencia entre las distancias no es significativa para los primeros cuatro puntos de medición. Los dos grupos de corazones presentaron mayor dispersión relativa en el punto 3 de medición (Tabla III).
Tabla I. Distancias (mm) entre las arterias coronarias y el anillo atrioventricular izquierdo en corazones fijados, en los cinco puntos de medición (P1-P5)

\begin{tabular}{lccccc}
\hline & \multicolumn{4}{c}{ Puntos de medición } \\
\hline Estadígrafos & P1 & P2 & P3 & P4 & P5 \\
n & 28 & 28 & 23 & 26 & 28 \\
Promedio & 4,36 & 5,42 & 6,13 & 5,26 & 6,50 \\
Desv. Estándar & 0,81 & 1,88 & 2,80 & 1,79 & 1,59 \\
\hline
\end{tabular}

Tabla II. Distancias (mm) entre las arterias coronarias y el anillo atrioventricular izquierdo en corazones frescos, en los cinco puntos de medición (P1-P5).

\begin{tabular}{lccccc}
\hline & \multicolumn{5}{c}{ Puntos de medición } \\
\cline { 2 - 6 } Estadígrafos & P1 & P2 & P3 & P4 & P5 \\
\hline $\mathrm{n}$ & 30 & 30 & 26 & 20 & 30 \\
Promedio & 3,83 & 4,69 & 6,27 & 5,98 & 10,16 \\
Desv. Estándar & 1,18 & 1,84 & 3,45 & 3,02 & 4,39 \\
\hline
\end{tabular}




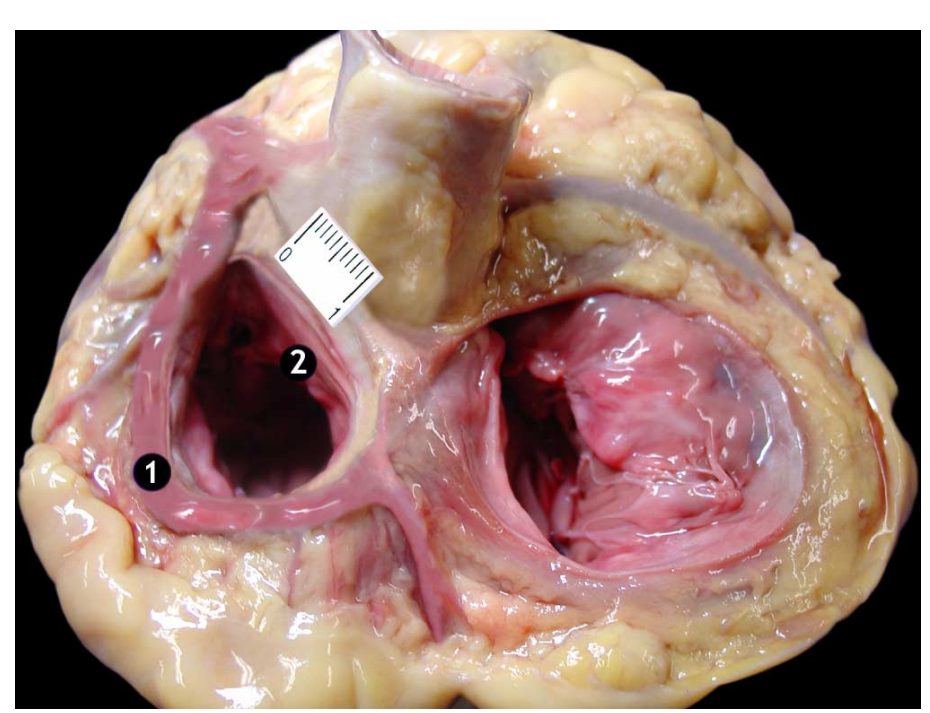

Fig. 4. Dominancia coronaria izquierda en corazón humano fresco. 1. Rama circunfleja de la arteria coronaria izquierda; 2. Valva Mitral; 3. Arteria coronaria derecha.

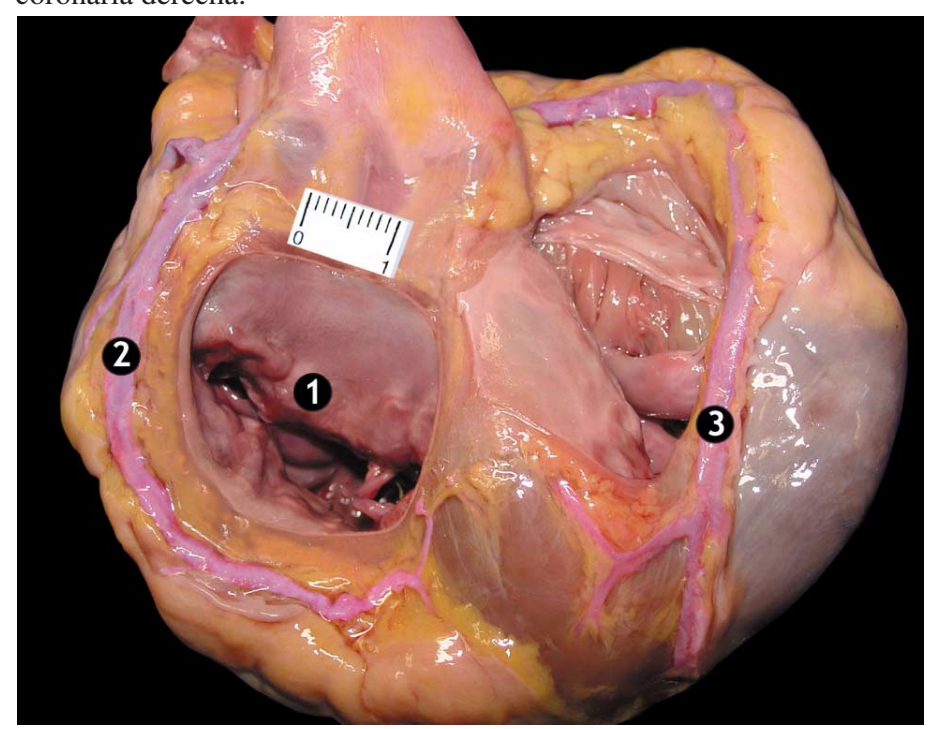

Fig. 5. Dominancia coronaria balanceada en corazón humano fresco. 1. Valva Mitral; 2. Rama circunfleja de la arteria coronaria izquierda; 3. Arteria coronaria derecha.

\section{DISCUSIÓN}

El conocimiento y la identificación del tipo de dominancia coronaria representan gran interés clínico y quirúrgico, debido a que variaciones en esa irrigación ocasionan diferentes grados de gravedad en casos de obstrucción (Lima Junior et al., 1993).

Se acepta ampliamente que el tercio proximal de la rama circunfleja está en riesgo de lesión iatrogénica durante las operaciones quirúrgicas de la valva mitral.

El tipo de dominancia coronaria y el grado de daño del anillo atrioventricular son factores importantes que contribuyen a esta complicación. La dominancia izquierda y codominancia se encuentran asociadas a una mayor proximidad de la rama circunfleja al anillo atrioventricular izquierdo, lo que otorga una mayor predisposición a una lesión por sutura durante un reemplazo y/o plastía valvar.

En corazones con dominancia coronaria izquierda, se observó en el punto 1 una distancia promedio menor respecto de los demás puntos de medición, con un promedio de 3,8 mm para los corazones fijados, y de 3,0 mm en corazones frescos. Nuestro resultado difiere levemente de la serie estudiada por Virmani et al., quienes obtuvieron un promedio de $4.1 \mathrm{~mm}$ a nivel de la comisura anterior (P1) en corazones con dominancia coronaria izquierda.

La dominancia coronaria derecha, en corazones fijados como frescos, presentó la menor distancia promedio en el punto 1 , con valores de 4,5 $\mathrm{mm}$ y 4,2 $\mathrm{mm}$, respectivamente. Estos datos concuerdan con los resultados obtenidos por Pessa et al. (2004) que señalaron para este punto una distancia de $3.99 \mathrm{~mm}$. Sin embargo, nuestros resultados revelan discordancia con otros autores, que señalaron una distancia entre estas estructuras de 8,0 y 8,4 mm, respectivamente (Cornu et al.; Virmani et al.).

Tabla III. Diferencias de las distancias promedio entre corazones frescos y fijados, para cada punto de medición.

\begin{tabular}{rcc|c|c}
\hline $\begin{array}{c}\text { Puntos de } \\
\text { Medición }\end{array}$ & $\begin{array}{l}\text { Dist. Promedio }(\mathrm{mm}) \\
\text { (Corazones Fijados) }\end{array}$ & $\begin{array}{l}\text { Dist. Promedio }(\mathrm{mm}) \\
\text { (Corazones Fresc os) }\end{array}$ & $\begin{array}{l}\text { Diferencia } \\
\text { Distancia }\end{array}$ & Valor p \\
\hline 1 & 4,36 & 3,83 & 0,53 & $0,0543+$ \\
2 & 5,42 & 4,69 & 0,73 & $0,1397+$ \\
4 & 6,13 & 6,27 & 0,14 & $0,8788+$ \\
5 & 5,26 & 5,78 & 0,72 & $0,3183+$ \\
\hline
\end{tabular}

+: No significativo *: Significativo al $5 \%$ 
PRADENAS, I.; HENRIQUEZ, J. \& OLAVE, E. Relaciones biométricas del anillo atrioventricular izquierdo y las arterias coronarias en el Hombre. Int. J. Morphol., 27(3):683-689, 2009.

Tabla IV. Distancias (mm) entre las arterias coronarias y el anillo atrioventricular izquierdo en corazones fijados, según dominancia coronaria, en cinco puntos de medición (P1 - P5)

\begin{tabular}{|c|c|c|c|c|c|c|}
\hline \multirow[b]{2}{*}{ Dominancia coronaria } & \multirow[b]{2}{*}{ Estadígrafos } & \multicolumn{4}{|c|}{ Puntos de medición } & \multirow[b]{2}{*}{ P5 } \\
\hline & & $\mathrm{P} 1$ & $\mathrm{P} 2$ & P3 & $\mathrm{P} 4$ & \\
\hline \multirow{6}{*}{ Derecha } & $\mathrm{n}$ & 21 & 21 & 16 & 19 & 21 \\
\hline & Mínimo & 3,0 & 3,6 & 3,7 & 2,7 & 3,9 \\
\hline & Máximo & 5,7 & 11,5 & 11,6 & 8,6 & 11,7 \\
\hline & Promedio & 4,5 & 5,9 & 7,1 & 5,5 & 6,5 \\
\hline & Desv. Estándar & 0,76 & 1,91 & 2,86 & 1,8 & 1,80 \\
\hline & Rango & 2,7 & 7,9 & 7,9 & 5,9 & 7,8 \\
\hline \multirow{6}{*}{ Izquierda } & $\mathrm{n}$ & 5 & 5 & 5 & 5 & 5 \\
\hline & Mínimo & 2,8 & 3,3 & 3,0 & 2,3 & 5,4 \\
\hline & Máximo & 5,1 & 5,3 & 5,4 & 5,5 & 7,4 \\
\hline & Promedio & 3,8 & 3,9 & 4,0 & 3,7 & 6,4 \\
\hline & Desv. Estándar & 0,85 & 0,82 & 0,90 & 1,19 & 0,85 \\
\hline & Rango & 2,3 & 2,0 & 2,4 & 3,2 & 2,0 \\
\hline \multirow{6}{*}{ Balanceada } & $\mathrm{n}$ & 2 & 2 & 2 & 2 & 2 \\
\hline & Mínimo & 3,5 & 3,5 & 3,7 & 6,2 & 6,1 \\
\hline & Máximo & 4,6 & 5,1 & 4,3 & 7,2 & 7,0 \\
\hline & Promedio & 4,0 & 4,3 & 4,0 & 6,7 & 6,5 \\
\hline & Desv. Estándar & 0,76 & 1,10 & 0,41 & 0,65 & 0,62 \\
\hline & Rango & 1,1 & 1,6 & 0,6 & 1,0 & 0,9 \\
\hline
\end{tabular}

Tabla V. Distancias (mm) entre las arterias coronarias y el anillo atrioventricular izquierdo en corazones frescos, según dominancia coronaria, en los cinco puntos definidos.

\begin{tabular}{lllllll}
\hline \multirow{3}{*}{ Dominancia coronaria } & & \multicolumn{5}{c}{ Puntos de medición } \\
\cline { 3 - 6 } Derecha & Estadígrafos & P1 & P2 & P3 & P4 & P5 \\
& n & 19 & 19 & 15 & 10 & 19 \\
& Mínimo & 2,4 & 2,9 & 4,3 & 32 & 5,1 \\
& Máximo & 6,3 & 9,2 & 17,5 & 13,8 & 22,5 \\
& Promedio & 4,2 & 5,4 & 8,0 & 7,7 & 10,6 \\
& Desv. Estándar & 1,2 & 1,9 & 3,6 & 3,3 & 4,5 \\
& Rango & 3,9 & 6,3 & 13,2 & 10,6 & 15,4 \\
Izquierda & n & 5 & 5 & 5 & 5 & 5 \\
& Mínimo & 1,4 & 1,9 & 2,2 & 2,2 & 2,4 \\
& Máximo & 3,8 & 4,5 & 5,4 & 5,6 & 11,2 \\
& Promedio & 3,0 & 3,5 & 3,8 & 4,0 & 7,7 \\
& Desv. Estándar & 1,1 & 1,2 & 1,4 & 1,4 & 3,4 \\
Balanceada & Rango & 2,4 & 2,6 & 3,2 & 3,4 & 8,8 \\
& n & 6 & 6 & 6 & 5 & 6 \\
& Mínimo & 2,5 & 2,8 & 3,1 & 3,3 & 4,2 \\
& Máximo & 4,3 & 4,4 & 6,5 & 5,9 & 16.5 \\
& Promedio & 3,3 & 3,4 & 4,1 & 4,5 & 10,7 \\
& Desv. Estándar & 0,6 & 0,6 & 1,2 & 1,1 & 4,9 \\
& Rango & 1,8 & 1,6 & 3,4 & 2,6 & 12,3 \\
\hline
\end{tabular}


Para la dominancia coronaria balanceada, que se analizó sólo en corazones frescos, las menores distancias promedio las observamos en los puntos 1 y 2 con valores de 3,3 y 3,4 mm, respectivamente. Para Pessa et al., las menores distancias promedio se presentaron en el punto 1 (3,5 $\mathrm{mm})$ resultado con el que coincidimos, sin embargo, en el punto 2 la distancia registrada por nosotros difirió en más de 1,0 mm, ya que estos autores obtuvieron $4,5 \mathrm{~mm}$ para el punto 2. Por otra parte, Cornu et al., registraron una distancia promedio de 4,5 $\mathrm{mm}$ en el punto $1 \mathrm{y}$ de 3,5 para el punto 2 , siendo nuestro resultado para este punto coincidente con los últimos autores.

Al comparar la distancia promedio según el tipo de dominancia coronaria para los dos subgrupos de corazones estudiados, observamos que ésta, en los corazones fijados con dominancia derecha es mayor en todos los puntos con respecto a los corazones con dominancia izquierda, a excepción del punto 5, donde prácticamente coinciden. Sin embargo, la mayor diferencia se presentó en el punto 3, donde los corazones con dominancia derecha presentaron una distancia promedio 3,12 $\mathrm{mm}$ mayor que la dominancia izquierda.

La misma situación se observó en el caso de corazo- nes frescos, solo que esta diferencia compromete a los cincos puntos de medición. La dominancia derecha, para estos últimos, presentó mayores distancias promedio en los cuatro primeros puntos de medición respecto de la dominancia coronaria balanceada, coincidiendo sólo en el último punto de medición con 10,6 y 10,7 mm, respectivamente. Cornu et al. observaron que la dominancia coronaria derecha presenta mayor distancia entre las estructuras estudiadas respecto de la dominancia coronaria balanceada en los primeros tres puntos de medición, presentándose la mayor diferencia en las distancias de estos subgrupos en el punto 1.

Al comparar dominancia izquierda y codominancia se aprecia que esta última sólo presenta menor distancia promedio en el segundo punto de medición.

Nuestra investigación muestra que los corazones frescos con dominancia derecha presentan mayor distancia promedio entre las estructuras estudiadas, seguramente a causa de la retracción de los corazones fijados. La menor distancia promedio entre la rama circunfleja y el anillo atrioventricular izquierdo se presenta, mayoritariamente, a nivel de la comisura anterior (P1), tanto en corazones frescos como fijados, con dominancia coronaria izquierda y co-dominancia

PRADENAS, I.; HENRIQUEZ, J. \& OLAVE, E. Biometric relationships between the left atrioventricular ring and the coronary arteries in man. Int. J. Morphol., 27(3):683-689, 2009.

SUMMARY: The relationships between the coronary arteries and the atrioventricular rings are important in cases of substitution or plastic of the corresponding valve. The vicinity of the circumflex branch of the left coronary artery to the left atrioventricular ring, at level of the previous corner, it has brought surgical complications, in some cases with fatal results. Due to this, we consider necessary to study the relationships biometric between the coronary arteries and the left atrioventricular ring. We use 58 hearts, 28 fixed hearts of Chilean individuals and 30 fresh hearts of Brazilian individuals, of ages between 18 and 84 years, of both sexes and without apparent heart surgery. The region of both coronary sulcus was dissected and we classified the pieces according to the type of coronary dominance. Later on, the atrium were dried up leaving visible the atrioventricular rings and their relationship with the coronary arteries. Measurement of the distances were made (in $\mathrm{mm}$ ) of the coronary arteries and their branches, in relation to the left atrioventricular ring. Five points settled down in the later portion of the ring, distributed in having felt anti-timetable. The points 1 and 5 located at level of the previous and later corners, respectively. The smallest distance between the branches of the coronary arteries and the left atrioventricular ring was presented in hearts with left coronary dominance, and it was of 3,8 $\mathrm{mm}$ (fixed hearts) and 3,0 $\mathrm{mm}$ (fresh hearts).

KEY WORDS: Anatomy; Heart; Coronary artery; Left atrioventricular ring; Valvar surgery.

\section{REFERENCIAS BIBLIOGRÁFICAS}

Biscegli, F.; Demarchi, V.\& Monteiro, R. Anatomía cardíaca. Bases morfológicas relevantes para o diagnostico e tratamento das Cardiopatias. Socesp. Cardiología, Atheneu, 2:8-16, 1996.

Cabrol, C. \& Christides C. Disposition habituelle et nomenclature des arteres coronaries. Bulletin de l'a Association des Anatomistes, 60(171): 21-25, 1976.
Cornu, E. ; Lacroix, PH. ; Christides, C. \& Laskar M Coronary artery damage during mitral valve replacement. J. Cardiovasc. Surg., 36(3):261-4, 1995.

Danielson, G,; Cooper, E. \& Tweddale, D. Circumflex coronary artery injury during mitral valve replacement. Ann. Thorac. Surg., 4:53-9, 1967. 
PRADENAS, I.; HENRIQUEZ, J. \& OLAVE, E. Relaciones biométricas del anillo atrioventricular izquierdo y las arterias coronarias en el Hombre. Int. J. Morphol., 27(3):683-689, 2009.

Du Plesssis, LA. \& Marchand, P. The anatomy of the mitral valve and its associated structures. Thorax, 19: 221-7, 1964

Lima Júnior R.;Cabral, R.H. \& Prates N.E.V.B Tipos de circulação e predominância das artérias coronárias em corações de brasileiros. Rev. Brás. Cir. Cardiovasc. 8(1): 9-19, 1993

Pessa, C.; Gomes, W.; Catani, R.; Catani, R; Prates, J.C. \& Buffolo, E. Anatomical relashionship between the posterior mitral valve annulus and the coronary arteries: implications to operative treatment. Braz. J. Cardiovasc Surg., 19(4):372-7, 2004.

Schlessinger, M. Relation of the anatomic pattern to pathologic conditions of the coronary arteries. Archives of Pathology, 30:403-15, 1940.

Seccombe, J. \& Schaff, H. Mitral valve repair: current techniques and indications. In: Franco, K. \& Verrier, E. Advanced therapy in cardiac surgery. London, England, 1999.

Tavilla, G. \& Pacini, D. Damage to the circunflex coronary artery during mitral valve repair whith sliding leaflet technique. Ann. Thorac. Surg., 66:291-4, 1998.

Testut, L \& Latarjet, A. Tratado de Anatomía Humana. Salvat, 2:97-108, 1960. V 2.

Virmani, R.; Patrick, K.C.; Parker, J. \& Mcallister, H.A. Suture obliteration of the circumflex coronary artery in three patients undergoing mitral valve operation. $J$. Thorac. Cardiovasc. Surg., 84:773-8, 1982.
Dirección para correspondencia:

Prof. Mg. Cs. Ilse Pradenas Mera

Unidad de Anatomía

Facultad de Ciencias de la Salud

Universidad de Antofagasta

CHILE

Email: ipradenas@uantof.cl

Recibido : 22-04-2009

Aceptado: 18-05-2009 
